\title{
Modelos Cosmológicos Quânticos em Minisuperespaço: Uma Revisão através do caso de Radiação
}

\author{
F. G. Alvarenga ${ }^{1}$, R. Fracalossi ${ }^{2}$ e S. V. de B. Gonçalves ${ }^{3}$ \\ Universidade Federal do Espírito Santo, ES, Brasil \\ E. V. C. Silva ${ }^{4}$ e G. A. Monerat ${ }^{5}$ \\ Universidade do Estado do Rio de Janeiro, RJ, Brasil \\ G. de O. Neto ${ }^{6}$ \\ Universidade Federal de Juiz de Fora, MG, Brasil \\ 1flavio.alvarenga@ufes.br 2rfracalossi@gmail.com 32sergio.vitorino@pq.cnpq.br \\ ${ }^{4}$ evasquez@uerj.br 5 germano.monerat@pq.cnpq.br \\ 6ilneto@ufjf.br
}

\begin{abstract}
Resumo
Neste trabalho propõe-se uma prescrição para formulação de modelos cosmológicos quânticos que descrevam a evolução do Universo Primordial. Tomando como exemplo um simples modelo de Universo sem curvatura espacial preenchido com fluido de radiação, aplica-se os passos necessários a elaboração de tais modelos, a saber: formalismo hamiltoniano da Relatividade Geral; condições de contorno e esquemas de interpretação da Mecânica Quântica.
\end{abstract}

\section{Introdução}

A inexistência de uma teoria completa de gravitação quântica implica na necessidade de se testar os efeitos quânticos em diferentes regimes e modelos. Neste sentido, modelos cosmológicos quânticos são simples exemplos nos quais as ideias da gravitação quântica podem ser aplicadas, por descreverem a possibilidade de nascimento do Universo, por exemplo, via um processo de tunelamento quântico, e assim evitar a singularidade inicial: es- tado de tamanho zero e densidade de energia infinita que constituem problemas inerentes ao estudo da evolução temporal do Universo. A chamada cosmologia quântica [1] surge então como uma solução alternativa a este problema. Além disso, a partir do estado inicial é possível estabelecer as condições que irão determinar a evolução posterior do Universo até que o regime clássico seja alcançado.

O processo de quantização ocorre no cenário de minisuperespaço, no qual con- 


\section{Blucher Proceedings IX Encontro Científico de Física Aplicada Blucher}

gelamos um número infinito de graus de liberdade e efetuamos a quantização dos graus remanescentes. DeWitt [2] foi quem primeiro adotou este esquema de quantização que hoje é largamente utilizado com diferentes conteúdos materiais e simetrias. Apesar disso existem problemas estruturais na teoria como a não explícita presença de uma variável do tipo tempo. Uma alternativa a essa questão reside na introdução fenomenológica de variáveis dinâmicas associadas a diferentes conteúdos materiais do Universo, com a pressão sendo expressa em termos de potencias de velocidade e uma dessas variáveis exercendo o papel do tempo. Este é o chamado formalismo de Schutz [3].

Neste trabalho revisa-se um modelo cosmológico em que o Universo está preenchido com um fluido perfeito barotrópico de radiação, como exemplo, para a implementação dos passos da prescrição da Cosmologia Quântica. O fluido fornece graus de liberdade para a introdução de uma variável tipo tempo. Isso permite transformar a equação de Wheeler-DeWitt em uma genuína equação de Schrödinger. A função de onda do Universo e o seu fator de escala do Universo serão determinados e analisados pelas interpretações da mecânica quântica de vários mundos e de de Broglie - Bohm.

\section{O Formalismo}

A formulação hamiltoniana da Relatividade Geral desenvolvida por Arnowitt, Deser e Misner, tornou-se conhecida como formalismo ADM. A dinâmica é revelada como a evolução de uma hipersuperfíe tridimensional, na qual os campos estão definidos. Parte-se da ação de Einstein-Hilbert com o termo de contorno:

$$
S_{g}=\int_{M} d^{4} x \sqrt{-g} R+2 \int_{\partial M} d^{3} x \sqrt{h} K,
$$

onde $R$ é o escalar de curvatura, tomado como função of $g^{\mu \nu}$ e suas derivadas, $K=h_{a b} K^{a b}$ tal que $K_{a b}$ é a curvatura extrínseca e $h_{a b}$ é a métrica induzida sobre a hipersuperfíe espacial tridimensional. Acima $\partial M$ é o contorno da variedade quadridimensional $M$ e $G$ é a constante gravitacional Newtoniana.

Para o Universo homogêneo e isotrópico adota-se a métrica na forma de Friedmann-Robertson-Walker $(\operatorname{com} c=1$ e $k=0)$ :

$$
d s^{2}=-N(t)^{2} d t^{2}+a(t)\left(d r^{2}+r^{2} d \Omega\right),
$$

em que $N(t)$ é a função lapso, que corresponde à componente normal da quadrivelocidade, $a(t)$ é o fator de escala do Universo. A inserção da métrica reduz a ação gravitacional a forma

$$
S_{g}=\int d t\left(-6 \frac{\dot{a}^{2} a}{N}\right),
$$

a qual mediante o formalismo canônico determina a hamiltoniana gravitacional:

$$
\mathscr{H}_{g}=-\frac{p_{a}^{2}}{24 a} .
$$

O Universo está totalmente preenchido por um fluido perfeito de radiação com a dinâmica descrita pelo formalismo de Schultz. Este formalismo emprega a representação da quadrivelocidade do fluido em termos de potenciais escalares $\phi, \theta$ e $S$ :

$$
U_{v}=\frac{1}{\mu}\left(\phi_{, v}+\theta S_{, v}\right),
$$

cada um satisfazendo sua própria equação de movimento. Na expressão acima $\mu$ é 


\section{Blucher Proceedings IX Encontro Científico de Física Aplicada Blucher}

a entalpia específica, $S$ é a entropia específica. As variáveis $\phi$ e $\theta$ não têm significado físico claro.

A ação da matéria é especialmente simples:

$$
S_{f}=\int d^{4} x \sqrt{-g} p,
$$

onde $p$ é a pressão, a qual está associada com a densidade de energia através da equação de estado $p=\frac{1}{3} \rho$. As equações de Einstein são obtidas pela variação da ação (6) mais a ação de Einstein-Hilbert em relação ao tensor métrico, enquanto variações relativas aos potenciais de velocidade fornecem as equações Eulerianas da evolução do fluido.

As relações termodinâmicas assumem a forma

$$
\rho=\rho_{0}(1+\Pi) ; \mu=(1+\Pi)+\frac{p}{\rho_{0}}
$$

$$
T_{s} d S=d \Pi+p d\left(\frac{1}{\rho_{0}}\right),
$$

sendo $\Pi$ a energia interna específica, $T_{S}$ a temperatura e $\rho_{0}$ a densidade de massa de repouso do fluido.

Escrevendo

$$
\begin{aligned}
d \Pi+p d\left(\frac{1}{\rho_{0}}\right) & =(1+\Pi) d[\ln (1+\Pi)+ \\
& \left.-\frac{1}{3} \ln \rho_{0}\right],
\end{aligned}
$$

identifica-se $T_{S}=1+\Pi$ e $S=\ln (1+$ П) $/ \rho_{0}{ }^{\frac{1}{3}}$.

Após algumas manipulações matemáticas, a pressão reduz-se a

$$
p=\frac{27}{256} \mu^{4} e^{-3 S} .
$$

A condição de normalização $U^{v} U_{v}=$ -1 permite expressar $\mu$ em termos dos potenciais:

$$
\mu=\frac{1}{N}(\dot{\phi}+\theta \dot{S}) .
$$

A inserção de (10) e (11) na ação da matéria (6), estabelece a Lagrangiana do fluido:

$$
\mathscr{L}_{f}=\frac{27}{256}\left(\frac{a}{N}\right)^{3}(\dot{\phi}+\theta \dot{S})^{4} e^{-3 S}
$$

Os momentos canônicos conjugados do fluido seguem da Lagrangiana acima,

$$
\begin{aligned}
p_{\phi} & =\frac{27}{64}\left(\frac{a}{N}\right)^{3}(\dot{\phi}+\theta \dot{S})^{3} e^{-3 S} \\
p_{S} & =\theta p_{\phi} \\
p_{\theta} & =0
\end{aligned}
$$

de modo a fornecer pelo formalismo canônico a Hamiltoniana do fluido

$$
\mathscr{H}_{f}=\frac{p_{\phi}^{\frac{4}{3}}}{a} e^{S} .
$$

As transformações canônicas

$$
\begin{aligned}
& T=p_{S} e^{-S} p_{\phi}{ }^{-\frac{4}{3}} ; p_{T}=p_{\phi}{ }^{\frac{4}{3}} e^{S} ; \\
& \bar{\phi}=\phi-\frac{4}{3} \frac{p_{S}}{p_{\phi}} ; \bar{p}_{\phi}=p_{\phi} .
\end{aligned}
$$

permitem colocar a Hamiltoniana (14) em uma forma mais sugestiva:

$$
\mathscr{H}_{f}=\frac{p_{T}}{a} .
$$

Finalmente, (4) e (16) compõem a Hamiltonina total clássica do sistema gravitacional acoplado à matéria:

$$
\mathscr{H}=-\frac{p_{a}^{2}}{24 a}+\frac{p_{T}}{a} .
$$




\section{A Equação de Wheeler- DeWitt e as Condições de Contorno}

O procedimento de quantização consiste na aplicacção dos operadores $\hat{p}_{a} \rightarrow-i \frac{\partial}{\partial a}$ e $\hat{p}_{T} \rightarrow-i \frac{\partial}{\partial T}$ em 17) de modo a impor a equação de Wheeler-DeWitt, $\hat{\mathscr{H}} \Psi=0$, sobre a função de onda do Universo. Para o modelo de radiação, esta equação assume a forma

$$
-\frac{1}{24} \frac{\partial^{2} \Psi}{\partial a^{2}}=i \frac{\partial \Psi}{\partial t}
$$

Ressalta-se que a interpretação da variável $t=-T$ como o tempo e da equação (18) como uma genuína equação de Schrödinger, requer que o correspondente operador

$$
\hat{\mathscr{H}}=-\frac{1}{24} \frac{\partial^{2}}{\partial a^{2}}
$$

seja auto-adjunto. $\mathrm{O}$ fator de escala $a$ está restrito ao domínio $a>0$, tal que a quantização no minisuperespaço diz respeito somente as funções de onda definidas no semi-eixo $(0, \infty)$. Em tais circunstâncias deve-se impor certas condições de contorno sobre as funções de onda permitidas que garantam a autoadjuntice de (19). Neste modelo isso é efetudo com a escolha do produto interno

$$
(\psi, \phi)=\int_{0}^{\infty} \psi^{*}(a) \phi(a) d a
$$

com domínio do operador restrito àquelas funções de onda que obdeçam à condição

$$
\left.\frac{\partial \Psi(a, t)}{\partial t}\right|_{a=0}=0 \text {. }
$$

Segue então, uma busca por soluções estacionárias da equação (18), ou seja, soluções do tipo

$$
\Psi(a, t)=e^{-i E t} \psi(a),
$$

onde $E$ é um parâmetro real. Assim sendo, a equação para $\psi(a)$ torna-se

$$
\frac{d^{2} \psi}{d a^{2}}+24 E \psi=0 \text {. }
$$

A solução geral da equação (23) é

$$
\psi_{E}(a)=C_{1} \cos \sqrt{24 E}+C_{2} \operatorname{sen} \sqrt{24 E},
$$

entretanto a condição (21) requer $C_{2}=$ 0 . Tal solução tem norma infinita, mas soluções de norma finita podem ser construídas pela superposição das mesmas. Assim sendo, as soluções gerais da equação de Wheeler-DeWitt (18) resultam de combinações lineares contínuas

$$
\Psi(a, t)=\int_{0}^{\infty} C(E) e^{-i E t} \psi_{E}(a) .
$$

Redefinindo $r^{2}=24 E$ e $f(r)=\frac{r}{12} C(E)=$ $\frac{r}{12} C\left(\frac{r^{2}}{24}\right)$, a escolha de $f(r)=e^{-\gamma r^{2}}$ ( $\gamma$ é uma constante real positiva), obtém-se:

$$
\begin{aligned}
\Psi(a, t) & =\int_{0}^{\infty} \cos (r a) e^{-\left(\gamma+\frac{i t}{24}\right) r^{2}} d r \\
& =\frac{\sqrt{\pi}}{2} \frac{e^{-\frac{a^{2}}{4\left(\gamma+\frac{i t}{24}\right)}}}{\sqrt{\gamma+\frac{i t}{24}}}
\end{aligned}
$$

\section{Interpretações}

É de fundamental importância o esquema de interpretação a ser adotado, uma vez que o sistema Universo não pode ser analisado pela tradicional interpretação de Copenhagen, que exige um observador externo, algo impossível se a função de onda descreve o Universo como um 


\section{Blucher Proceedings \\ IX Encontro Científico de Física Aplicada Blucher}

todo. Como alternativa propõe-se efetuar as predições cosmológicas atavés das interpretações de vários mundos e de de Broglie - Bohm [4].

\subsection{Vários Mundos}

$\mathrm{Na}$ interpretação de vários mundos na medição não existe colapso da função de onda, mas simplesmente a divisão do mundo em muitos mundos, cada um deles tendo seu próprio resultado de medição. $\mathrm{O}$ valor esperado do fator de escala pode ser calculado da maneira usual

$$
\begin{aligned}
& \langle a\rangle_{t}=\frac{\int_{0}^{\infty} \Psi^{*}(a, t) a \Psi(a, t) d a}{\int_{0}^{\infty} \Psi^{*}(a, t) \Psi(a, t) d a} \\
& =\frac{\Gamma(1)}{24 \Gamma\left(\frac{1}{2}\right)} \sqrt{576 \gamma^{2}+t^{2}} .
\end{aligned}
$$

O Universo é não singular e assintoticamente correspondente ao modelo clássico, quando $t \rightarrow \infty 1$

\subsection{De Broglie- Bohm (dBB)}

$\mathrm{Na}$ interpretação $\mathrm{dBB}$ um sistema físico individual compreende uma onda propagando-se no espaço e tempo juntamente com uma partícula que movese continuamente guiada pela onda. A onda é matematicamente descrita por $\Psi(a, t)=R(a, t) e^{i S(a, t)}$, a qual é uma solução da equação (18). A trajetória $a(t)$ é postulada por:

$$
p_{a}=\left.\frac{\partial S(a, t)}{\partial a}\right|_{a=a(t)},
$$

com o movimento dirigido pela equação de Hamilton-Jacobi:

$$
\frac{\partial S}{\partial t}+\frac{1}{24}\left(\frac{\partial S}{\partial a}\right)^{2}+V+Q=0
$$

onde $Q$ é o chamado potencial quântico:

$$
Q=-\frac{1}{24 R} \frac{\partial^{2} R}{\partial a^{2}} .
$$

Neste modelo sem termo de curvatura, o potencial clássico $V$ é nulo.

A amplitude da onda $R(a, t)$ e a fase $S(a, t)$ são identificadas em $(26)$ :

$$
\begin{gathered}
R(a, t)=\frac{\sqrt{\pi}}{2\left(\gamma^{2}+\frac{t^{2}}{576}\right)^{\frac{1}{4}}} e^{-\frac{\gamma a^{2}}{4\left(\gamma^{2}+\frac{t^{2}}{576}\right)}} ; \quad(31) \\
S(a, t)=\frac{a^{2} t}{96\left(\gamma^{2}+\frac{t^{2}}{576}\right)}+\operatorname{tg}^{-1}\left(-\frac{t}{24 \gamma}\right) .
\end{gathered}
$$

O momento $p_{a}$ pode ser obtido da equação $\dot{a}=\frac{\partial N \mathscr{H}}{\partial p_{a}}$, ou seja, $p_{a}=-12 \dot{a}$. Portanto, este resultado inserido em (28) possibilita a determinação da trajetória bohmiana,

$$
a(t)=a_{0} \sqrt{576 \gamma^{2}+t^{2}}
$$

onde $a_{0}$ é uma constante de integração arbitrária positiva. Esta solução, evidentemente não singular, exibe a mesma dependência temporal do valor esperado (27).

O potencial quântico, calculado inserindo (31) em (30), é dado por:

$$
Q(a, t)=-\frac{\gamma}{48\left(576 \gamma^{2}+t^{2}\right)^{2}}\left[288 \gamma a^{2}-\right.
$$

$$
\left.\left(576 \gamma^{2}+t^{2}\right)\right]
$$

\footnotetext{
${ }^{1}$ No tempo cósmico $a \propto t^{\frac{1}{2}}$, o que é revelado neste modelo em termos do tempo conforme adotado $d t^{\prime}=a d t$, ou seja, $N(t)=a(t)$.
} 


\section{Blucher Proceedings \\ IX Encontro Científico de Física Aplicada Blucher}

Ao potencial quântico está associada uma força

$$
F(a, t)=-\left.\frac{\partial Q(a, t)}{\partial a}\right|_{a=a(t)} \propto \frac{1}{a^{3}},
$$

a qual possui um caráter repulsivo. Existe uma força repulsiva atuando de modo a evitar a singularidade acerca da região $a=0$.

\section{Conclusões}

Rubakov e Lapchinskii [5] efetuaram inicialmente a quantização em minisuperespaço para um modelo cosmológico de Universo preenchido por um fluido de radiação empregando o formalismo de Schutz, sem efetuarem previsões cosmológicas acerca da evolução do fator de escala do Universo. Lemos [6] calculou o valores esperado para o fator de escala, via interpretação de vários mundos, através de propagadores quânticos. Tal modelo foi confirmado por Pinto Neto [7] e colaboradores utilizando a interpretação dBB. Em 2002, Fabris e colaboradores [8] generalizaram os modelos cosmológicos isotrópicos quânticos para diferentes conteúdos materiais. Neste trabalho revisou-se tais procedimentos de quantização com uma construção mais simplificada de pacote de onda, destacando o caráter não singular das soluções cosmológicas nos esquemas de interpretação de vários mundos e dBB.

\section{Agradecimentos}

Os autores agradecem à comissão organizadora do IX Encontro Científico de Física Aplicada pela excelência do evento.

\section{Referências}

[1] J. J. Halliwell, Quantum Cosmology and Baby Universes. (World Scientific, Singapore, 1991).

[2] B. S. DeWitt, Phys. Rev. 160, 1113 (1967).

[3] F. G. Alvarenga, R. G. Furtado e S. V. B. Gonçalves, S. Brazilian Journal of Physics, v. 47, p. 96 (2016)

[4] N. Pinto Neto. Teorias e Interpretações da Mecânica Quântica. São Paulo, Livraria da Física (2010).

[5] V. G. Lapchinskii e V. A. Rubakov, Theo. Math. Phys. 33. 1076 (1977).

[6] N. A. Lemos, J. Math. Phys. 37, 1449 (1996).

[7] J. Acacio de Barros, N. Pinto-Neto e M. A. Sagioro-Leal, Phys. Lett. A 241, 229 (1998).

[8] F. G. Alvarenga, J. C. Fabris, N. A. Lemos, G. A. Monerat, Gen. Rel. Grav. 34, 651 (2002). 